\title{
Separate weighing of male and female broiler breeders by electronic platform weigher using camera technologies
}

\author{
Dong Liu ${ }^{\mathrm{a}, \mathrm{c}}$, Erik Vranken ${ }^{\mathrm{a}, \mathrm{b}}$, Gijs van den Berg ${ }^{\mathrm{b}}$, Lenn Carpentier ${ }^{\mathrm{a}}$, Alberto Peña Fernández ${ }^{\mathrm{a}}$, \\ Dongjian $\mathrm{He}^{\mathrm{c}}$, Tomas Norton ${ }^{\mathrm{a}, *}$ \\ ${ }^{\text {a } M 3-B I O R E S, ~ K U ~ L e u v e n, ~ L e u v e n, ~ B e l g i u m ~}$ \\ ${ }^{\mathrm{b}}$ Fancom BV, Panningen, NL-5980, the Netherlands \\ ${ }^{\mathrm{c}}$ College of Mechanical and Electronic Engineering, Northwest A\&F University, Yangling 712100, China
}

\section{A R T I C L E I N F O}

\section{Keywords:}

Precision Livestock Farming

Broiler breeder

Weighing

Image processing

\begin{abstract}
A B S T R A C T
The body weight of breeding broiler chickens (broiler breeders) is an important control variable used to optimize the amount, quality and fertility of the eggs being laid. In modern breeding barns, the population of animals generally supports a female:male ratio of 10:1, wherein males and females each receive their own food supply. To control this supply the broiler breeders are weighed using automatic electronic platform weighers and the weights are then classified into cockerel and hen categories using theoretical growth curves. However, due to the non-uniform growth of the animals and replacement (called spiking) of cockerals this classification is not always correct, causing a risk of under/over feeding of the population. To overcome this challenge, in this study we have developed a system that integrates the electronic platform weigher with the low-cost 3D Kinect camera was employed to separate weigh male and female broiler breeders under commercial conditions. A novel image processing algorithm is proposed. The algorithm first constructed the Height Accumulating Image (HAI) using depth image to locate the region of interest (ROI) where a broiler breeder jumps onto the weigher, then the comb size was calculated on RGB image as the gender classifying feature, and finally, an adaptive classification threshold was determined by the kernel density estimation using the recent days comb size measurements. The results showed that enough measurements can be obtained for estimating overall weight expectation with the average acceptance rate of $77.32 \%$. Based on these accepted measurements, the accuracy, sensitivity, precision, and specificity were $99.7 \%, 98.82 \%, 100 \%$ and $100 \%$ respectively.
\end{abstract}

\section{Introduction}

The broiler industry is the process by which broiler chickens are reared and prepared for meat consumption. Due to the high feed conversion ratio ( $1.5 \mathrm{~kg}$ of feed to produce $1 \mathrm{~kg}$ of meet), the modern commercial strains of broilers provide high-quality, low-fat, and lowcost animal protein that meet the requirement of population growth. Being upstream of the broiler supply chain, broiler breeder farms have the objective to maximise the number of good-quality, fertile eggs and hatched broiler chicks (Mench, 2017). The parent stock clearly possess the fast-growing traits of broiler birds themselves. In order to limit problems, such as obesity, lameness, and ascites, and maintain adequate reproductive capabilities, broiler breeders are feed restricted, mainly through control the weight throughout the rearing and breeding period (Widowski and Torrey, 2017). There is an obvious conflict of interest to ensure proper growth and development with maximum egg production.
In a commercial breeding barn, the sexes are mixed with a ratio of $8-10$ hens to one cockerel and it is better to feed separately because of their different growth rates and nutrition requirements (Silveira et al., 2014; Uğurlu et al., 2017). Farmers aims to precise measure daily weight gain of cockerels and hens separately, then give feedback on corresponding feed supply, ultimately make them follow their own empirical growth curve accurately during a feeding cycle. Currently, farmers can randomly sample a certain number of cockerels and hens, then calculate the mathematical expectation of the overall level which is labor intensity and also stressful for birds (Amraei et al., 2017; Mortensen et al., 2016). An alternative solution is the usage of the precision weighing system (Chedad, et al., 2000; Ren et al., 2020), through which the bird can freely access the platform to trigger the systems' step response. The body weight of each visitor is recorded by calculating the response amplitude, and the daily weight gain of the flock is estimated subsequently. However, Chedad et al. (2003) reported that the flock weight 


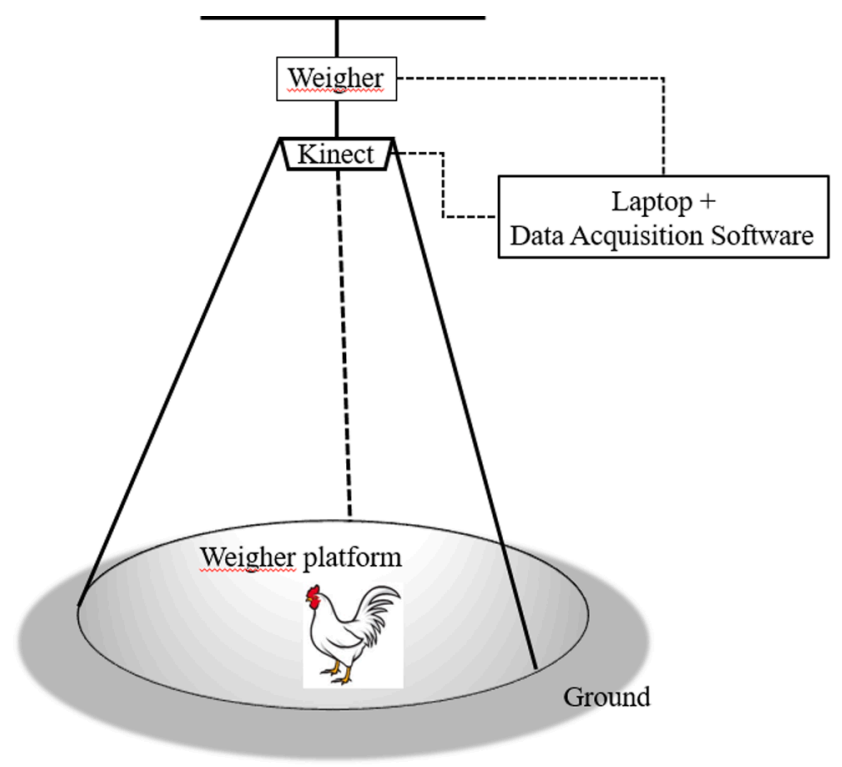

Fig. 1. The experimental equipment includes a Kinect V2 camera and an automatic poultry weighing system.

was underestimated by $30 \%$ since heavier birds visited the weigher less frequently than others, especially at the end of the breeding cycle. To solve this problem, Vranken et al. (2005) combined the weigher with image analysis to correct the bias to an average of $5 \%$ at the end of the fattening period. Cangar et al. (2006) minimized the error to an average of $0.14 \%$ using a time-varying prediction model, in which cumulative feed intake as the process input. Although the improved system can accurately estimate the flock weight distribution, it is still unable to distinguish sexes. Currently, the gender is roughly classified by a threshold based on the assumption that cockerels are heavier than hens. Due to non-uniform growth, the overlapping of cockerels and hens' weight distributions is very common in commercial breeding barns, especially when the older cockerels are replaced by younger ones to improve fertility levels, which will lead to a further increase in the estimation error.

The advancement of computer vision offers non-intrusive approaches to estimate live animal weight indirectly with promising application aspects, for example pigs (Schofield, 1990; Kashiha et al., 2014), cows (Tasdemir et al., 2011; Liu et al., 2020), and sheep (Menesatti et al.,2014). Some studies also attempted to estimate poultries' body weight by computer vision technologies (De Wet et al., 2003). For example, in the experimental scenarios, Mollah et al. (2010) used body surface to estimate body weight with the error ranged from $0.04 \%$ to $16.47 \%$. In the commercial production scenarios, Mortensen et al. (2016) developed a 3D camera-based system to estimate the weight of free-ranging broilers. The watershed algorithm was used on depth image to separate individual broiler from the group, then a combination of 12 geometric features (1D, 2D and 3D) were extracted and used for prediction with the error of $7.8 \%$. The camera-based systems have an obvious advantage in its ability to weigh several birds at once but the accuracy is not good enough and limits their application in commercial farms. This is mainly because: 1) The high-density breeding and the similar appearance bring challenges to segment individuals from the group by image processing technologies; 2) Any changes in the body posture can cause the fleecy feathers to deform, leading to the distortion of geometric features (e.g. flapping wings, squeezing each other). Because of this, there is no camera-based poultry weighing system for commercial applications, and the precision weighing system (Doyle and Leeson, 1989; Lokhorst, 1996) is still the mainstream approach accepted by the farm managers.

As to the analysis above, the existing automatic approaches lack a

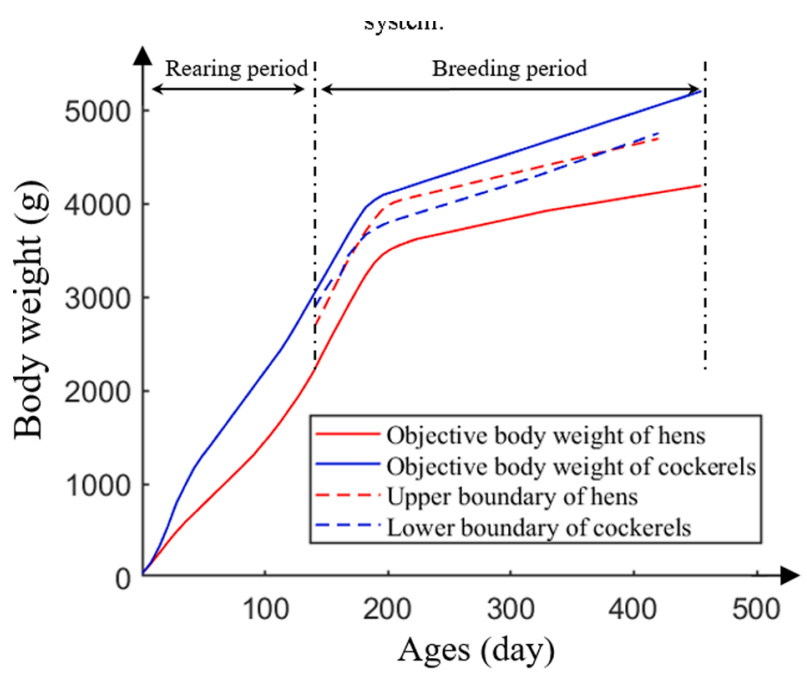

Fig. 2. The rearing and breeding period of broiler breeders and the expected body weight. The data collection was carried out in the pre- and postbreeding period.

desirable solution to precise monitor gender-based broiler breeders' body weight. This study aims to provide a solution that can simultaneously obtain individual body weight and gender tag in the commercial barn. To achieve this objective, the precision weighing system was used to obtain the body weight and the low-cost Kinect camera was integrated into the original system which provide the gender tag (Supplementary video).

\section{Materials and methods}

\subsection{Experimental protocol and equipment}

An overview of the experimental equipment is shown in Fig. 1, in which the RGB-D images were acquired by a Kinect camera (Version 2, Microsoft Corp., Redmond, WA) and the body weights were acquired by an automatic poultry weighing system (LUMINA 47, FANCOM BV, Netherlands). The weighing system was installed on the ceiling at a distance of $190 \mathrm{~mm}$ off the hard cement ground and the Kinect V2 was installed on the scale pole to obtain top-view images. The distance from the camera to the ground is $1.5 \mathrm{~m}$. In order to make the acquired dataset cover the entire breeding period as much as possible, the data collection was carried out twice in the pre- and post-breeding period (Fig. 2). The first part dataset was collected on 300-day-old broiler breeders and the second part dataset was collected on 150-day-old broiler breeders in Netherlands. Both of the two farms are typical breeding barn contained approximately 48,000 Ross 308 with a gender ratio of 10:1 (hens: cockerels). Each experiment lasts 7 days, from 4 h00 to 17 h00 each day. Kinect camera recorded calibrated color stream and depth stream with 15FPS. The sampling frequency of the weighing system is $10 \mathrm{~Hz}$.

\subsection{Dataset description and labelling}

The dataset consists of RGB images, depth images and corresponding weight data sequence. RGB images are mapped to the depth image coordinate reference with the resolution of $512 * 424$ pixels using a Kinect toolbox for Matlab (Terven and Córdova-Esparza, 2016). Since the imaging system and weighing system are working independently with different sampling frequency in this study, manual synchronization is essential in order to obtain consistent information. The synchronization process is illustrated in Fig. 3, includes the following steps:

(a) Recording body weight readings. Stable measurements were selected from the raw weighting data sequence by detecting step signals, which were triggered by one bird jumping on the weigher. This process 


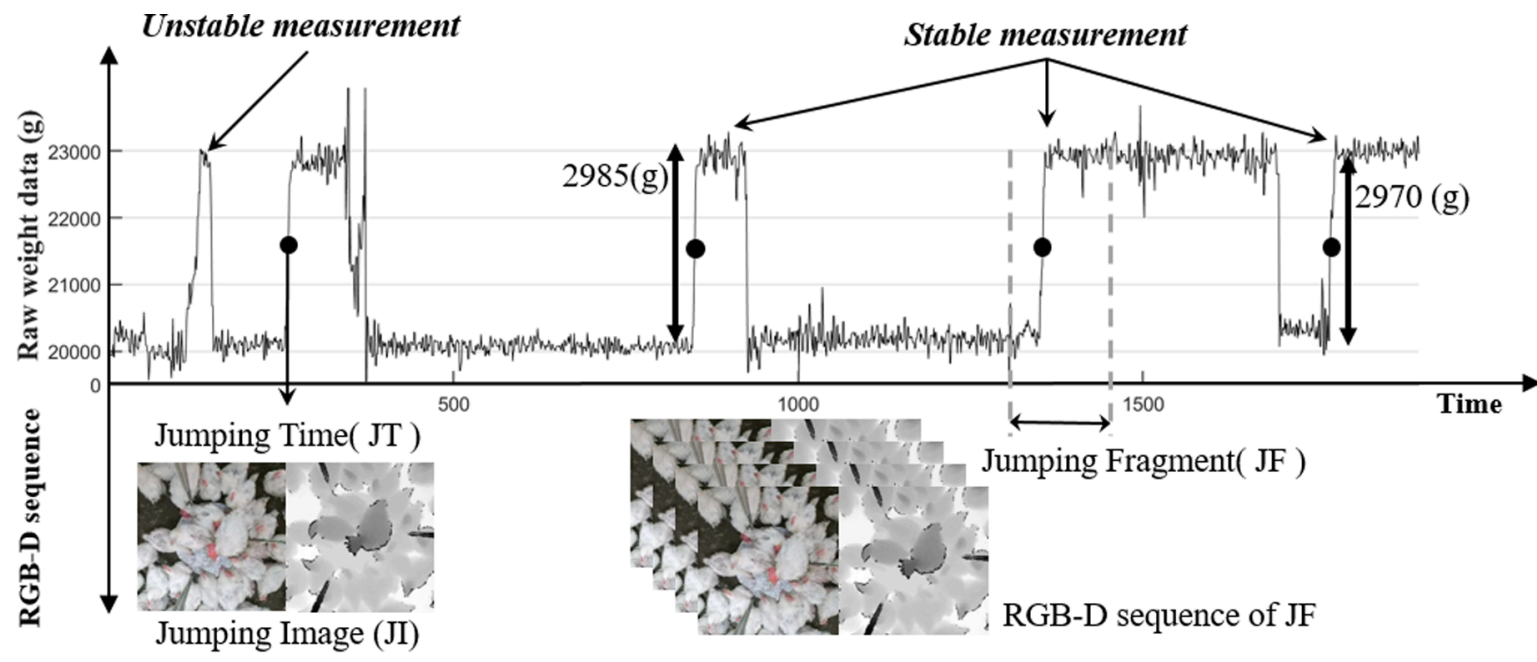

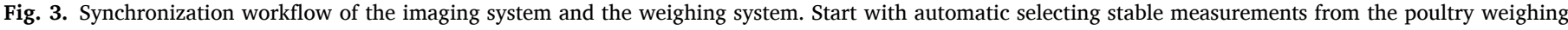

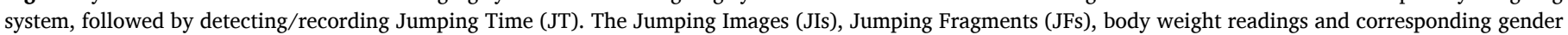
tags were recorded to form the dataset.

Table 1

Summary of data sets.

\begin{tabular}{lll}
\hline Age & Gender & Number of broiler breeders jumped onto the weigher \\
\hline \multirow{2}{*}{ 300 days } & Cockerel & 22 \\
& Hen & 537 \\
150 days & Cockerel & 55 \\
& Hen & 237 \\
\hline
\end{tabular}

\begin{tabular}{|c|c|}
\hline \multicolumn{2}{|c|}{ Loading labeled data sets } \\
\hline Jumping Time (JT) & Jumping Fragment (JF) \\
\hline \multicolumn{2}{|c|}{ Locating ROI } \\
\hline $\begin{array}{l}\text { Binary Jumping } \\
\text { Image }(\mathrm{JI})\end{array}$ & $\begin{array}{l}\text { Height Accumulating } \\
\text { Image (HAI) }\end{array}$ \\
\hline \multicolumn{2}{|c|}{ The intersection operation } \\
\hline \multicolumn{2}{|c|}{$---\frac{-}{\text { Measuring comb size }}--\frac{\searrow--}{-}--$} \\
\hline RGB images of JF & ROI mask \\
\hline \multicolumn{2}{|c|}{ The intersection operation } \\
\hline \multicolumn{2}{|c|}{ Update by the latest measurements } \\
\hline \multicolumn{2}{|c|}{ Dataset of comb size } \\
\hline \multicolumn{2}{|c|}{ 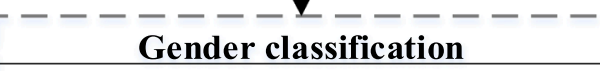 } \\
\hline \multicolumn{2}{|c|}{ Adaptive threshold of comb size } \\
\hline The kernel smooth & g function estimation \\
\hline
\end{tabular}

Fig. 4. The overall framework of proposed method to obtain gender tag using image processing algorithms.

was performed automatically by the automatic poultry weighing system (LUMINA 47), in which the Jumping Time (JP) and corresponding weight reading were recorded (as shown by the black dot in Fig. 3), and unstable measurements was excluded (as shown in Fig. 3).

(b) System synchronization. Since the sampling rate of the weighing system and image system is different, it is necessary to manually synchronize the two systems. Based on these recorded
Jumping Time in step (a), the corresponding Jumping Images (JI) are manually selected from the RGB-D image sequence.

(c) Clipping the jumping fragment. The first 15 frames and the last 15 frames of each JI were extracted from video sequence as Jumping fragment (JF). These 30 frames (two second) are the most important period since the jump action happened within this period.

(d) Recording the gender. The gender of each bird who jumped onto the weigher was also manually recorded for final algorithm verification.

With the above data synchronization process, 559 stable measurements of broiler breeders at the age of 300 days and 291 stable measurements at the age of 150 days were labeled which took $70 \mathrm{~h}$ of human labor. A summary of the number of broiler breeders and the gender in the data sets can been seen in Table 1 .

\section{Methodology}

The method starts with analyzing weight data sequence to provide reliable body weight readings. Then, a series of image processing algorithms are proposed to get the gender tag of each body weight reading. The image processing algorithm consists three steps: (1) A region of interest (ROI) localization algorithm is performed on depth image to locate where a bird jump onto the weigher; (2) A comb size measuring algorithm is applied to the color image within the extracted ROI. The comb size is taken as the gender feature; (3) A gender thresholding model is designed based on the comb feature. The overall framework is shown in Fig. 4 and the details are introduced in Section 3.1, Section 3.2, and Section 3.3, respectively.

\subsection{Locating the ROI mask in the depth image}

Due to the natural behavior of clustering habit (e.g. crowding around the weigher and pecking the platform), it's a big challenge to separate the individual from the group (Mortensen et al., 2016). Instead of segmenting individuals from the group, we proposed an alternative solution aimed to find out the ROI where the bird jumped on the weigher. Four image processing steps were designed to locate the ROI based on assumptions: In a Jumping Fragment, the most significantly changing area is where the jumping action takes place; Compared with other possible actions (e.g. free walking, running), the jumping action is characterized as frame-wise rising in the vertical (height) direction.

a) Manually select the initial circular ROI, the radius of which is 1.5 times of the weigher platform. In the depth image (Fig. 5a), the initial 


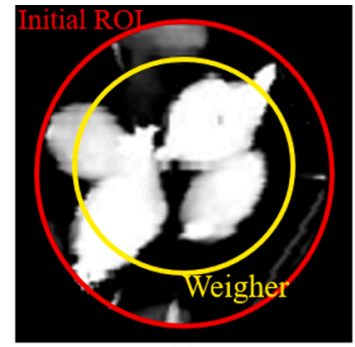

(a)

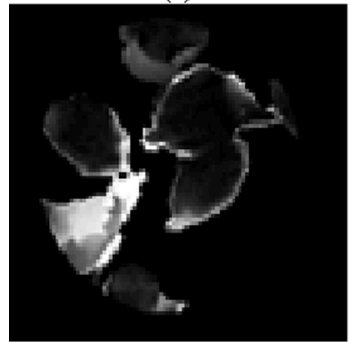

(d)

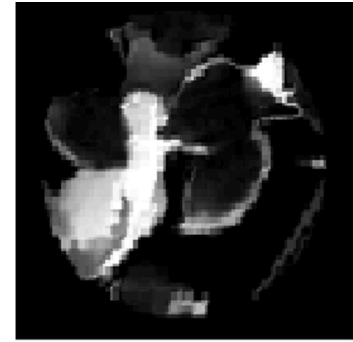

(b)

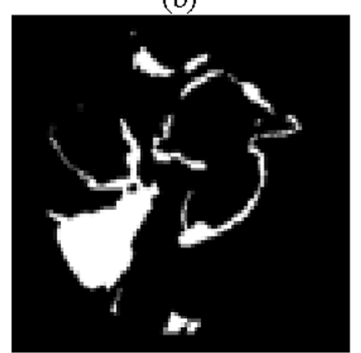

(e)

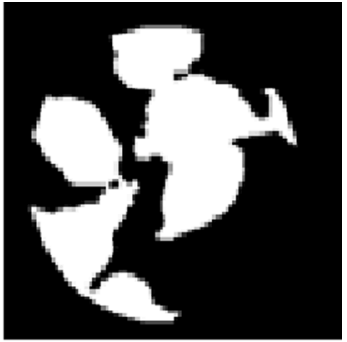

(c)

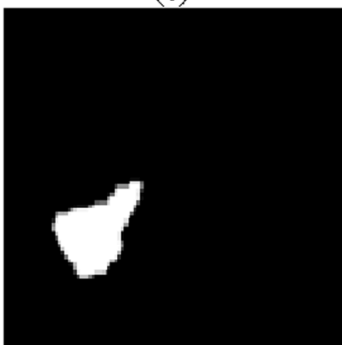

(f)

Fig. 5. An example of locating the region of interest by depth image analysis. (a) The original depth image; (b) Height Accumulating Image; (c) Binarized Jumping Image; (d) The intersection of HAI and binary JI; (e) The output after binarization of figure d; (f) The final ROI mask.

ROI is inside the red circle and the weigher platform is inside the yellow circle, and different gray pixels represent different heights. Since the camera is mounted on the scale pole which is fixed to the weigher, the swing of the weigher will not cause ROI changes;

b) To quantify the jumping action pattern (frame-wise rising), the Height Accumulating Image (HAI) was designed, which was calculated

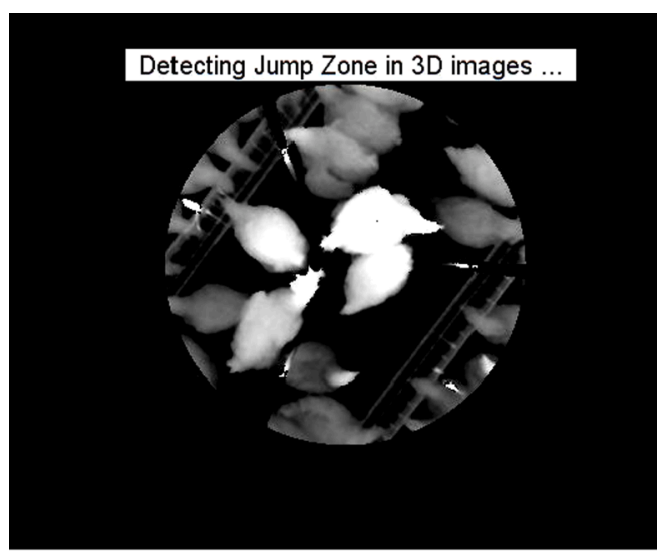

(a)
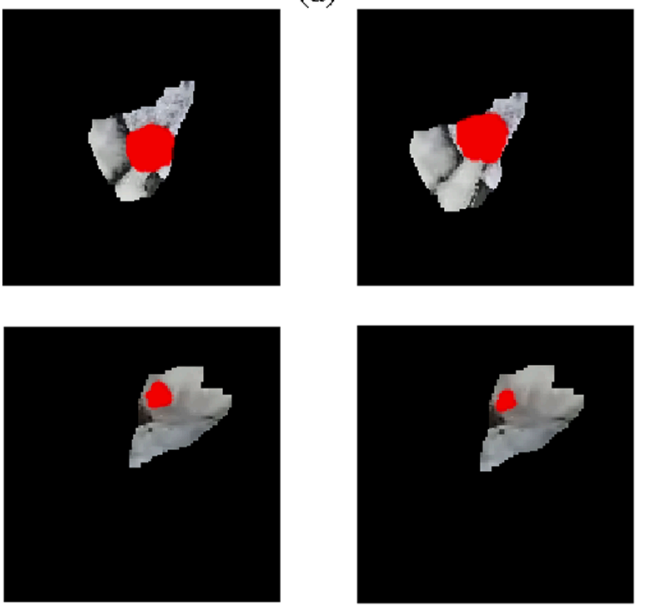

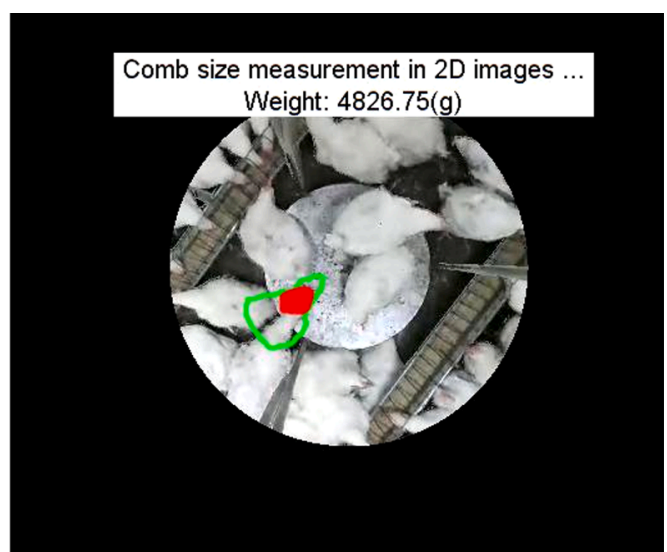

(b)
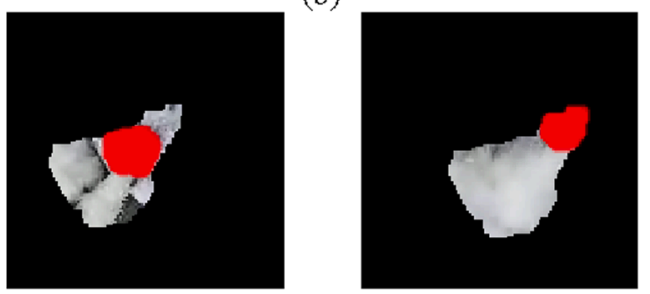

(c)
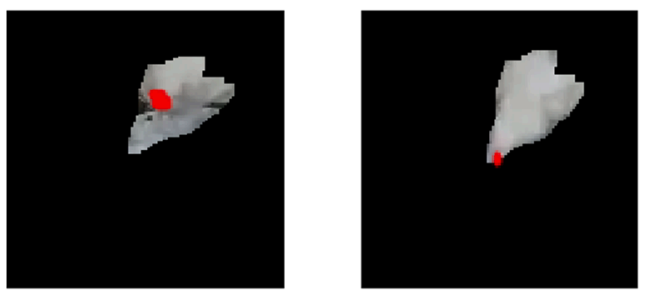

(d)

Fig. 6. The results of comb size detection on a cockerel and a hen. (a) Detecting the Jumping Zone in depth image; (b) Measuring the comb size in RGB image; (c) Cockerel: the outputs were 406 pixels, 392 pixels, 412 pixels and 362 pixels respectively. The final output is the maximum value 412 pixels; (d) Hen: the ouputs were 53 pixels, 43 pixels, 40 pixels and 3 pixels respectively. The final output is the maximum value 53 pixels. 
by Formula (1)-(3). Firstly, the frame difference $F D(t)$ was obtained by Formula (1); Then, pixels with ascending change pattern were preserved and pixels with drop mutations were eliminated by formula (2). Pixels with abnormal ascending changes were also eliminated regarding as noises, because height changes in adjacent frames cannot exceed the bird's body height (The threshold Tin Formula (2)); At last, the HAI was constructed by Formula (3). Fig. 5b shows an example of HAI, in which a cockerel jumped onto the weigher from the lower left corner. It's obvious that pixels with ascending change are highlighted.

$F D_{t}(x, y)=I_{t}(x, y)-I_{t-1}(x, y), t \in[1,30]$

Where, FD is frame difference within one Jumping Fragment. $x$ is the row number.yis the column number. tis the frame number.

$H_{t}(x, y)=\left\{\begin{array}{l}F D_{t}(x, y), \text { if } 0<F D_{t}(x, y)<T \\ 0, \text { if } F D_{t}(x, y) \leqslant 0 \mid F D_{t}(x, y)>T\end{array}\right.$

Where, $T$ is the mean body height of broiler breeder which is measured in pixels. In this study, we conducted a pre-experiment under the experimental conditions and determined that the body height of broiler breeders is in the range of $[120,220]$ pixels. Any value of $T$ within this range is possible, because subsequent steps will further accurately locate the ROI.

$H A I=\sum_{t=1}^{30} H_{t}(x, y)$

c) In addition to the jumping action, the highlighted area also has noises from other birds movement, as shown in Fig. 5b. To further eliminate these noises, a prior knowledge was used. That is, at the Jumping Time, when one leg steps upon the platform, around half body has reached inside of the weigher. Therefore, in the binarized Jumping Image (Fig. 5c), The final ROI must be within the white area. Then, the intersection of the HAI (Fig. 5b) and the Jumping Image (Fig. 5c) was calculated, as shown in Fig. 5d and Fig. 5e (The output after binarization). It can be seen that noise area generated by the movement of other broiler breeders in the HAI was suppressed.

d) At last, an erosion operation followed by an expansion operation were conducted in order to remove small noise. The largest connected area is extracted as the final ROI mask, as shown in Fig. $5 \mathrm{f}$.

\subsection{Measuring comb size from the RGB image}

An observational empirical is that the cockerel's comb size is much bigger and brighter than that of the hen. The comb is easily identified in RGB image due to its obvious color characteristics (bloody red color), a simple threshold in the R-channel can achievement satisfactory results, which can be quantified by Formula (4). Note that this simple method only works during the light period of the photoperiodic regime. The light intensity was approximately $25 \mathrm{~lx}$ in this study.

$\operatorname{Comb}(x, y)=\left\{\begin{array}{c}1, \text { if } R(x, y)-B(x, y)>10 \& R(x, y)-G(x, y)>10 \\ 0, \text { else }\end{array}\right.$

Where, R, G and B are the three channels of the color image.

Since the located ROI mask is the area where a jumping action occurred, the broiler breeder who visits the weigher must pass by this region. Based on this fact, RGB images in the corresponding Jumping Fragment are intersected with the ROI mask. The broiler breeder's comb will be exposed within the ROI and measured by Formula (4) subsequently. Some examples of the above procedures are shown in Fig. 6, in which a cockerel was jumped upon the weigher from the left down corner. The Jumping Zone (ROI mask) was obtained in the depth images (Fig. 6a) and was highlighted by the green line in Fig. 6b. The comb within the green line was detected (by Formula (4)) and identified as the one who visited the weigher. The comb size would be measured 30 times within a Jumping Fragment, and the maximum value is taken as the final output. The reason for using the maximum value is that there may be no comb exposed in some of these images. Examples of the cockerel and the hen were shown in Fig. $6 \mathrm{c}$ and Fig. $6 \mathrm{~d}$ respectively, in which outputs for the cockerel were $(406,392,412,362)$ pixels and outputs for the hen were $(53,43,40,3)$ pixels.

\subsection{Adaptive threshold for the gender classification}

Although the difference in terms of comb size between the cockerel and the hen is significant in Fig. 6c and Fig. 6d, gender classification with a fixed threshold cannot meet the actual requirement since the comb size grows gradually during a breeding cycle. To develop an adaptive threshold, the basic idea is that the threshold needs to be determined based on the recent measurements. In this study, the kernel density estimation (KDE) (DeVeaux et al., 1999) was used to determine such an adaptive threshold. In statistics, $\mathrm{KDE}$ is a non-parametric way to estimate the probability density function of a random variable. Since physiological data such as the comb size or the body weight are believed to follow a normal distribution, the Gaussian function is selected as the kernel function. The KDE can be explained as below:

Let $X=\left(x_{1}, x_{2}, \ldots, x_{n}\right)$ be the observed samples of broiler breeders' comb size, which from some distribution with an unknown density $f(x)$. Given that $X_{c}=\left(x_{c 1}, x_{c 2}, \ldots, x_{c n}\right)$ and $X_{h}=\left(x_{h 1}, x_{h 2}, \ldots, x_{h n}\right)$ be the labelled samples of cockerel and hen's comb size, which were drawn from distributions of $f_{c}(x)$ and $f_{h}(x)$ with Gaussian density, the shape of $f(X)$ can be estimated by its Gaussian kernel density estimator with Formula (5).

$\widehat{f}(x)=\frac{1}{n h} \sum_{i=1}^{n} G\left(\frac{x-x_{i}}{h}\right)$

Where Gis the Gaussian kernel, nis the number of observations, and $h>0$ is a smoothing parameter called Bandwidth, which reflects the overall flatness of the KDE curve. Note that the integral area under the $\widehat{f}(x)$ curve is 1 .

Since the cockerel has a bigger comb than the hen, an optimal comb size threshold Texists on the $\widehat{f}(x)$ curve, in which any measurement bigger than Tbelongs to the cockerel and vice versa. In the KDE curve of $\widehat{f}(x)$, the threshold $T$ is determined by Formula (6), where $R$ is the proportion of cockerel in the flock. In this study, the sexes are mixed with a ratio of ten hens to one cockerel, thus Requals to $1 / 11$.

$\int_{T}^{+\infty} \widehat{f}(x) d x=R$

\section{Results and discussion}

Applying the proposed algorithm (Section 3) to the dataset (Table 1), the results were evaluated by AcceptanceRate, Accuracy, Sensitivity, Precisionand Specificity. The definitions of these metrics were shown in Formula (7-11) respectively, in which theAcceptanceRate evaluated the accuracy of the image processing algorithm (Section 3.1 and Section 3.2) for obtaining the comb size and others evaluated the performance of the gender classification model (Section 3.3).

AcceptanceRate $=\frac{\text { acceptedmeasurements }}{\text { Totalnumberofmeasurements }}$

Accuracy $=\frac{\text { numberoftruepositivies }+ \text { numberoftruenegatives }}{\text { Acceptedmeasurements }}$

Sensitivity $=\frac{\text { numberoftruepositivies }}{\text { numberoftruepositivies }+ \text { numberoffalsenegatives }}$

Precision $=\frac{\text { numberoftruepositivies }}{\text { numberoftruepositivies }+ \text { numberoffalsepositives }}$

Specificity $=\frac{\text { numberoftruenegatives }}{\text { numberoftruenegatives }+ \text { numberoffalsepositives }}$ 
Table 2

Results of the image processing algorithm.

\begin{tabular}{clll}
\hline & 150-day-old & 300-day-old & Total \\
\hline Dataset (Table 1) & 292 (hen/ & 559 (hen/ & 851 (hen/ \\
& cockerel: 237/55) & cockerel: 537/22) & cockerel: 774:77) \\
Accepted & 196 (hen/ & 462 (hen/ & 658 (hen/ \\
measurements & cockerel: 150/46) & cockerel: 442/20) & cockerel: 592/66) \\
AcceptanceRate & $67.12 \%$ (hen/ & $82.65 \%$ (hen/ & 77.32\% (hen/ \\
& cockerel: 63.29\%/ & cockerel: $82.31 \%$ / & cockerel: 76.49\%/ \\
& $83.63 \%)$ & $90.91 \%)$ & $85.71 \%)$ \\
\hline
\end{tabular}

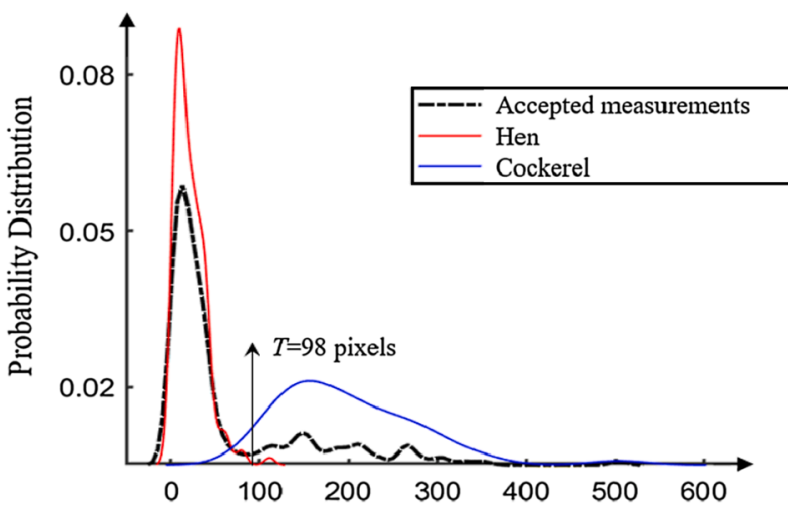

Comb size(In pixels): DataSize-196 (Cockerel-46/Hen-150)

(a)

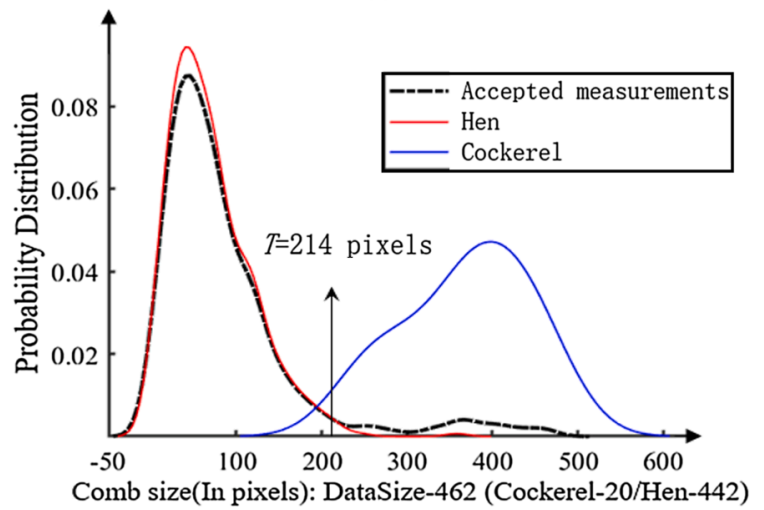

(b)

Fig. 7. The estimated distribution curve by KDE. (a) 150-day-old broiler breeders; (b) 300-day-old broiler breeders.

\subsection{Performance of the image processing algorithm}

The results were summarized in Table 2. For the 150-day-old broiler breeders, 196 out of 292 birds' comb were accurately obtained with anAcceptanceRateof $67.12 \%$; For the 300 -day-old broiler breeders, 462 out of 559 samples were accepted with an AcceptanceRate of $82.65 \%$. The main reason of these failure cases is that some broiler breeders jump onto the weigher in abnormal postures, such as flying onto the weigher, jumping with sideways, and two birds jumping at the same moment. For these special cases, the image processing algorithm (Section 3.1) also located a ROI, but no comb is exposed within this ROI (Section 3.2). As a result, the comb size output was zero and the corresponding measurement was rejected. Nevertheless, the overall acceptance rate of $77.32 \%$ can meet the application requirement.

Compared to the old broiler breeder (300-day-old), theAcceptanceRateof the young broiler breeder is much lower. This is because young birds are always more flexible and hyperactive than the older, resulting in more abnormal jumping action. Besides, it can be seen that more young cockerels were obtained than old cockerels, which is
Table 3

Performance of the gender classification model.

\begin{tabular}{llll}
\hline Metrics & 150 -day-old & 300-day-old & Total \\
\hline Accuracy & $99.5 \%$ & $99.8 \%$ & $99.7 \%$ \\
Sensitivity & $98.78 \%$ & $98.87 \%$ & $98.82 \%$ \\
Precision & $100 \%$ & $100 \%$ & $100 \%$ \\
Specificity & $100 \%$ & $100 \%$ & $100 \%$ \\
\hline
\end{tabular}

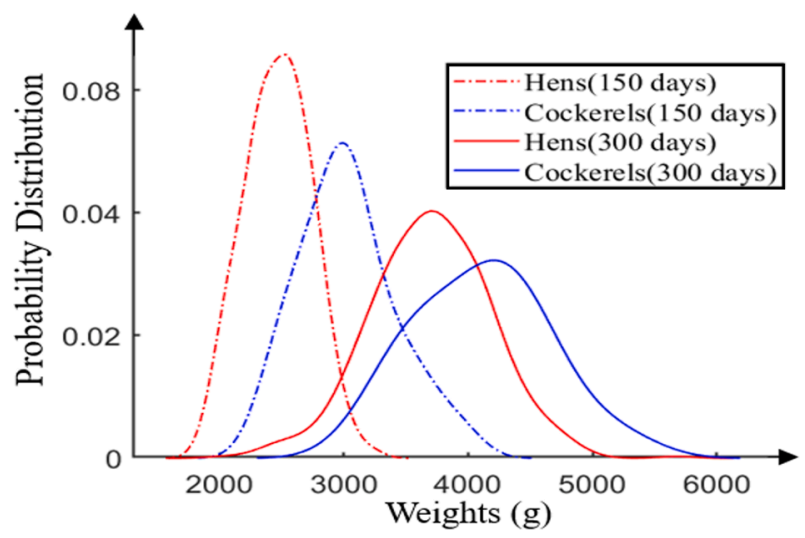

Fig. 8. The body weight distribution of hens and cockerels at different ages.

consistent with previous study (Chedad et al., 2003). That is, heavier birds visited the weigher less frequently, especially at the end of the breeding cycle.

\subsection{Performance of the gender classification model}

Applying KDE to those accepted measurements (Table 2), the estimated distribution curves

were shown in Fig. 7. The curves of hens (red solid line) and cockerels (blue solid line) approximately subject to Gaussian distribution. The KDE curve of overall accepted measurements was shown as a black dotted line. Integrating the KDE curves from right to left (according to Formula (6)), the threshold Tcan be determined as 98 pixels for 150-dayold broiler breeders and 214 pixels for 300-day-old broiler breeders.

The gender classification results were summarized in Table 3 . Once a measure was accepted (the comb size was successfully obtained), it had a $99.7 \%$ chance of being correctly classified. Replaying these misclassified video samples, seven hens were misclassified to the cockerel and none of the cockerels were misclassified. All misclassifications were caused by a comb from another cockerel breaking into the located ROI.

\subsection{Comparing with body weight distribution}

The body weight distributions of the dataset were shown in Fig. 8. Due to non-uniform growth, both young and old broiler breeders' body weight distributions were heavily overlapped indicating that it is not feasible to classify the gender by a body weight threshold. Comparing Fig. 7 and Fig. 8, the comb size is a much more robust gender feature since either young or old broiler breeders is distinguishable.

\subsection{Eliminating cockerels with poor physical conditions}

In a commercial breeding barn, some cockerels with low-sperm quality will be eliminated and replaced by young cockerels. Comparing the body weight distributions of 150-day-old cockerels (blue dotted line in Fig. 8) and 300-day-old hens (red solid line in Fig. 8), young cockerels is lighter than old hens indicating that most of young cockerels would be misclassified if the body weight was used as gender 


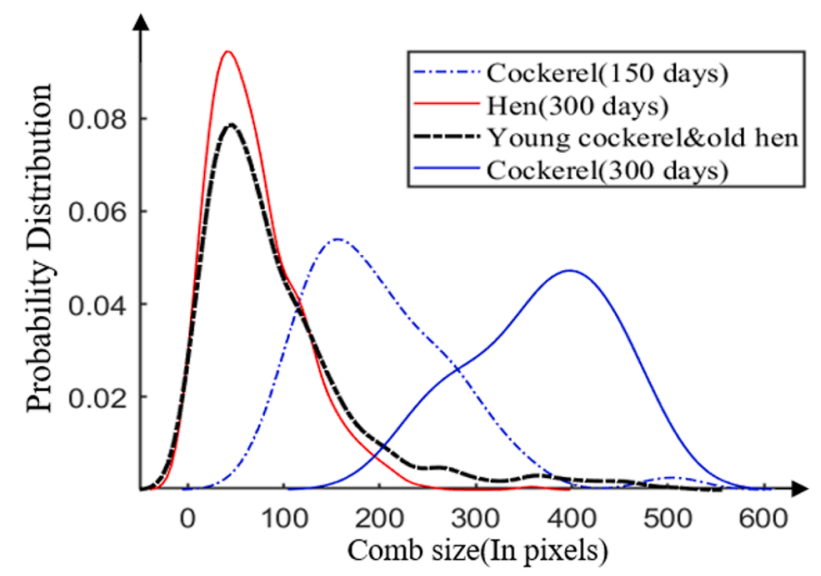

Fig. 9. The comb size distribution of the old hen and the young cockerel.

feature. It brings big challenges to the perspective of precision feeding.

As a contrast, Fig. 9 shows the comb size distributions of young cockerels and old hens, in which the distribution curves partially overlap, but most of young cockerels' comb are still bigger than that of old hens. It indicates that the gender classification accuracy will drop slightly at the beginning of the replacement, but the comb size distribution of the young cockerel will shift to right (shift from the blue dotted line to the blue solid line) quickly with the growth of cockerels. This is because the comb size of old hens will not grow a lot, but it grows fast for cockerels.

\subsection{Towards commercial application}

In this study, the platform weighing system and the 3D Kinect camera have different sampling rate $(10 \mathrm{~Hz}$ for the weigher and $15 \mathrm{~Hz}$ for the camera). The body weight data and image sequences were synchronized manually, which cost a large amount of labor work. Towards to commercial application, two systems must be integrated to obtain synchronized data stream. Besides, the proposed image processing algorithm to locate the ROI and obtain the comb size achieved an AcceptanceRateof $77.32 \%$ on average. It indicates less measurements can be obtained comparing to the poultry weighing system. In order to obtain enough measurements to estimate the overall expectations, more equipment needs to be deployed in the breeding barn.

\section{Conclusions}

This study proposed a non-intrusive system to separately weighing of male and female broiler breeder. The system integrates an electronic platform weigher with 3D Kinect camera which was developed and evaluated under commercial. The system proved that enough measurements can be obtained for estimating overall weight expectation with the average acceptance rate of $77.32 \%$. Then, an adaptive threshold for the gender classification model based on kernel density estimation was proposed, through which the accuracy, sensitivity, precision, and specificity achieved 99.7\%, 98.82\%, 100\% and 100\% respectively, which meet the requirement of commercial application.

\section{CRediT authorship contribution statement}

Dong Liu: Conceptualization, Methodology, Software, Validation, Formal analysis, Investigation, Data curation, Writing - original draft, Writing - review \& editing. Erik Vranken: Writing - review \& editing, Resources, Data curation, Supervision. Gijs den Berg: Data curation. Lenn Carpentier: Conceptualization, Formal analysis, Investigation, Writing - review \& editing. Alberto Peña Fernández: Conceptualization, Formal analysis, Investigation, Writing - review \& editing.
Dongjian He: Resources, Formal analysis, Writing - review \& editing, Supervision. Tomas Norton: Conceptualization, Methodology, Investigation, Writing - review \& editing, Supervision, Project administration.

\section{Declaration of Competing Interest}

The authors declare that they have no known competing financial interests or personal relationships that could have appeared to influence the work reported in this paper.

\section{Acknowledgements}

The authors thank the company Fancom BV for assistance and guidance with the experimental facilities and equipment.

\section{Appendix A. Supplementary material}

Supplementary data to this article can be found online at https://doi. org/10.1016/j.compag.2021.106009.

\section{References}

Amraei, S., Abdanan Mehdizadeh, S., Sallary, S., 2017. Application of computer vision and support vector regression for weight prediction of live broiler chicken. Eng. Agric. Environ. Food 10 (4), 266-271. https://doi.org/10.1016/j.eaef.2017.04.003.

Cangar, Ö., Aerts, J.M., Vranken, E., Berckmans, D., 2006. End-weight prediction in broiler growth. Br. Poult. Sci. 47 (3), 330-335. https://doi.org/10.1080/ 00071660600741735.

Chedad, A., Aerts, J.M., Vranken, E., Lippens, M., Zoons, J., Berckmans, D., 2003. Do heavy broiler chickens visit automatic weighing systems less than lighter birds? Br. Poult. Sci. 44 (5), 663-668. https://doi.org/10.1080/00071660310001643633.

Chedad, A., Vranken, E., Aerts, J.-M., Berckmans, D., 2000. Behaviour of chickens towards automatic weighing systems. IFAC Proc. 33 (29), 207-212. https://doi.org/ 10.1016/s1474-6670(17)36778-2.

De Wet, L., Vranken, E., Chedad, A., Aerts, J.M., Ceunen, J., Berckmans, D., 2003. Computer-assisted image analysis to quantify daily growth rates of broiler chickens. Br. Poult. Sci. 44 (4), 524-532. https://doi.org/10.1080/00071660310001616192.

Deveaux, R.D., Bowman, A.W., Azzalini, A., 1999. Applied smoothing techniques for data analysis. Technometrics 41, 263. https://doi.org/10.2307/1270572.

Doyle, I., Leeson, S., 1989. Automatic weighing of poultry reared on a litter floor. Can. J. Anim. Sci. 69 (4), 1075-1081. https://doi.org/10.4141/cjas89-122.

Kashiha, M., Bahr, C., Ott, S., Moons, C.P.H., Niewold, T.A., Ödberg, F.O., Berckmans, D., 2014. Automatic weight estimation of individual pigs using image analysis. Comput. Electron. Agric. 107, 38-44. https://doi.org/10.1016/j.compag.2014.06.003.

Liu, D., He, D., Norton, T., 2020. Automatic estimation of dairy cattle body condition score from depth image using ensemble model. Biosyst. Eng. 194, 16-27. https:// doi.org/10.1016/j.biosystemseng.2020.03.011.

Lokhorst, C., 1996. Automatic weighing of individual laying hens in aviary housing systems. Br. Poult. Sci. 37 (3), 485-499. https://doi.org/10.1080/ 00071669608417880.

Mench, J.A., 2017. Advances in Poultry Welfare. Woodhead Publishing. https://doi. org/10.1016/C2015-0-04880-8.

Menesatti, P., Costa, C., Antonucci, F., Steri, R., Pallottino, F., Catillo, G., 2014. A lowcost stereovision system to estimate size and weight of live sheep. Comput. Electron. Agric. 103, 33-38. https://doi.org/10.1016/j.compag.2014.01.018.

Mollah, M.B.R., Hasan, M.A., Salam, M.A., Ali, M.A., 2010. Digital image analysis to estimate the live weight of broiler. Comput. Electron. Agric. 72 (1), 48-52. https:// doi.org/10.1016/j.compag.2010.02.002.

Mortensen, A.K., Lisouski, P., Ahrendt, P., 2016. Weight prediction of broiler chickens using 3D computer vision. Comput. Electron. Agric. 123, 319-326. https://doi.org/ 10.1016/j.compag.2016.03.011.

Ren, G., Lin, T., Ying, Y., Chowdhary, G., Ting k $C$. 20)ก Aoricultıral rnhotics research applicable to poultry production: A revie https://doi.org/10.1016/j.compag.2020.

Schofield, C.P., 1990. Evaluation of image ana pigs. J. Agric. Eng. Res. 47, 287-296. htt 80048-Y.

Silveira, M.M., de Freitas, A.G., Moraes, C.A., Gomes, F.S., Litz, F.H., Martins, J.M.S., Fagundes, N.S., Fernandes, E.A., 2014. Feeding management strategy for male broiler breeders and its effects on body weight, hatchability and fertility. Brazil. J. Poultry Sci. 16 (4), 397-402. https://doi.org/10.1590/1516-635x1604397-402.

Tasdemir, S., Urkmez, A., Inal, S., 2011. Determination of body measurements on the Holstein cows using digital image analysis and estimation of live weight with regression analysis. Comput. Electron. Agric. 76 (2), 189-197. https://doi.org/ 10.1016/j.compag.2011.02.001.

Terven, J.R., Córdova-Esparza, D.M., 2016. Kin2. A Kinect 2 toolbox for MATLAB. Sci. Comput. Program. 130 (15), 97-106. https://doi.org/10.1016/j.scico.2016.05.009.

Uğurlu, M., Akdağ, F., Teke, B., Salman, M., 2017. Effects of protein in diet and sex ratio on egg production, egg and hatching chick weight, fertility, hatchability and 
embryonal mortality in pheasants (Phasianus Colchicus). Brazi. J. Poultry Sci. 19 (2), 231-238. https://doi.org/10.1590/1806-9061-2016-0423.

Vranken, E., Chedad, A., Aerts, J.M., Berckmans, D., 2005. Improving the accuracy of automatic broiler weighing by image analysis. Precis. Livestock Farm. 05, 265-271.
Widowski, T., Torrey, S., 2017. Rearing young birds for adaptability. In: Advances in

Poultry Welfare, pp. 49-76. https://doi.org/10.1016/B978-0-08-100915-4.00003-8. 